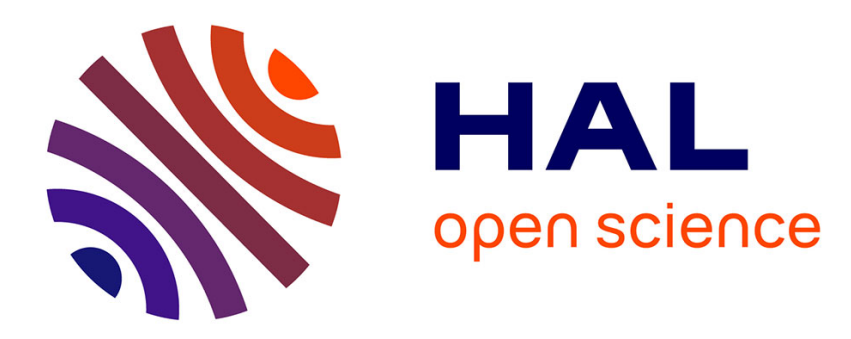

\title{
Possible observation of the roughening transition in helium
}

\author{
Sebastien Balibar, B. Castaing
}

\section{To cite this version:}

Sebastien Balibar, B. Castaing. Possible observation of the roughening transition in helium. Journal de Physique Lettres, 1980, 41 (14), pp.329-332. 10.1051/jphyslet:019800041014032900 . jpa-00231791

\section{HAL Id: jpa-00231791 https://hal.science/jpa-00231791}

Submitted on 1 Jan 1980

HAL is a multi-disciplinary open access archive for the deposit and dissemination of scientific research documents, whether they are published or not. The documents may come from teaching and research institutions in France or abroad, or from public or private research centers.
L'archive ouverte pluridisciplinaire HAL, est destinée au dépôt et à la diffusion de documents scientifiques de niveau recherche, publiés ou non, émanant des établissements d'enseignement et de recherche français ou étrangers, des laboratoires publics ou privés. 


\title{
Possible observation of the roughening transition in helium
}

\author{
S. Balibar and B. Castaing \\ Groupe de Physique des Solides de l'Ecole Normale Supérieure, 24, rue Lhomond, 75231 Paris 05, France
}

(Reçu le 3 mars 1980, accepté le 27 mai 1980)

\begin{abstract}
Résumé. - Nous proposons l'existence d'une transition rugueuse à la surface des cristaux hexagonaux d'hélium 4 à $1 \mathrm{~K}$. Nous justifions cette hypothèse grâce à des arguments à la fois théoriques et expérimentaux. Nous réinterprétons des mesures antérieures de la tension interfaciale liquide-solide. Enfin nous en déduisons une estimation de l'énergie de marche en-dessous de $1 \mathrm{~K}$.
\end{abstract}

\begin{abstract}
We suggest that the surface of hcp ${ }^{4} \mathrm{He}$ crystals undergoes a roughening transition at $1 \mathrm{~K}$. Both theoretical and experimental arguments are presented. Previous measurements of the liquid-solid interfacial tension are reinterpreted. An estimation of the step-energy below $1 \mathrm{~K}$ is deduced.
\end{abstract}

The theory of the roughening transition was first introduced by Burton, Cabrera and Franck [1] in order to explain different behaviours observed by metallurgists in the growth dynamics of crystals. Since this historical paper, many theoretical works [2] have been devoted to this problem, especially since 1973 when one realized that there is a duality with the Kosterlitz-Thouless transition in the planar $X Y$ model [3], and since numerical simulations were possible [4]. The roughening transition is now known as a very general term for a class of 2D order-disorder transitions, its theory being possibly applied to systems as various as crystal surfaces, 2D liquid helium films [5], 2D Coulomb systems [3], Bloch walls in ferromagnets, ferroelectric materials, spin glasses, etc.

However, there is very little evidence for the experimental observation of this transition. To our knowledge, only three systems exhibit a behaviour which has been explained in terms of these theories : the Kosterlitz-Thouless theory accounts for the onset of superfluidity in 2D He films [5], and for the crystallization of 2D electron systems [6]. The only observation concerning a solid surface is the experiment by Jackson and Miller [7] on the growth dynamics of plastic crystals. Unfortunately, in :his experiment the crystals are not at the thermodynamical equilibrium, which makes it difficult to test the roughening transition theory quantitatively.

We would like to reinterpret here previous measurements [8] of the liquid-solid ${ }^{4} \mathrm{He}$ interfacial tension in the light of more recent measurements which use different methods $[9,10]$. This new interpretation supposes the existence of a roughening transition at the surface of helium crystals, where atomically smooth facets existing at low temperature seem to disappear above $1 \mathrm{~K}$, leading to a completely rounded shape with a rough surface [9]. This permits us to interpret the discrepancy between the various measurements of the liquid-solid interfacial tension. We justify the existence of this transition theoretically and compare it to the prediction of delocalized quantum defects made recently by Andreev [11]. We then make the first experimental estimation of the energy for a step at the surface of a solid helium facet, and obtain finally some new details concerning the variation of the interfacial tension with the crystallographic angle. We think that the experiment by Balibar, Edwards and Laroche [8] (BEL) was the first observation of the static properties of a crystal which undergoes a roughening transition. Since a stable interface between liquid and solid ${ }^{4} \mathrm{He}$ is easy to obtain at the thermodynamical equilibrium around $1 \mathrm{~K}$, we hope that this system will permit one to test the theory of the roughening transition in the very near future.

In BEL's experiment [8], solid helium was forced, at the melting pressure, through a hole of radius $R$ and supposed to form there a rounded meniscus, a sort of solid bubble. The maximum pressure $\Delta p$ between the two sides of the solid bubble was measured, and a value for the liquid-solid interfacial tension $\alpha$ was deduced with the aid of the well-known Laplace law :

$$
\Delta p=\frac{2 \alpha}{R} \text {. }
$$


As shown on the figure, the values obtained were observed to jump around $1 \mathrm{~K}$ from a high temperature value of $\sim 0.14 \mathrm{erg} . \mathrm{cm}^{-2}$ up to a low temperature value of $\sim 1 \mathrm{erg} . \mathrm{cm}^{-2}$. The interface was generally observed to be liquid-like, and the meniscus was presumed to be always quasi-spherical. A tentative interpretation of the jump was to suppose the appearance at $1 \mathrm{~K}$ of a layer of bcc solid ${ }^{4} \mathrm{He}$ at the interface between liquid and hcp solid ${ }^{4} \mathrm{He}$. The true hcp solid-liquid interfacial tension was supposed to be that at low temperature, i.e. $1 \mathrm{erg} . \mathrm{cm}^{-2}$, and that of the bcc solid-liquid was $0.98 \mathrm{erg} . \mathrm{cm}^{-2}$. It was assumed possible to reduce the hcp surface energy, even at a temperature somewhat lower than the bcc to hcp transition temperature $(1.46 \mathrm{~K})$ by intercalating a bcc layer between the hcp and the liquid phase. One could then reasonably have thought that this layer would act as a seed for the nucleation of the bcc phase when the liquid + hcp solid was warmed up to $1.46 \mathrm{~K}$. Actually there is no convincing experimental evidence that the hcp phase can be superheated into the stability region of the bcc phase. However, by crossing $1.46 \mathrm{~K}$, we never observed the growth of the bcc phase from this interface, but from that between the liquid and the walls of the cell.

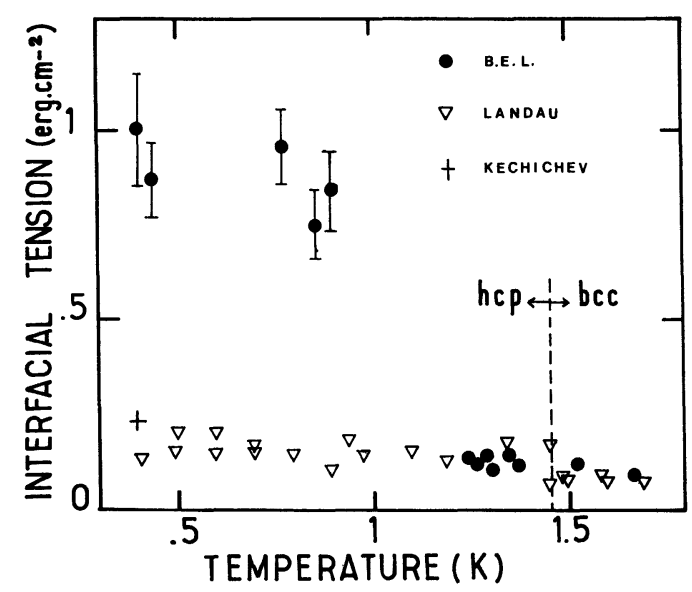

Fig. 1. - Liquid-solid ${ }^{4} \mathrm{He}$ interfacial tension $\alpha$ versus temperature $T$. At low temperature the measurements made by Landau and by Kechichev do not agree with the measurements of Balibar, Edwards and Laroche (BEL). The discrepancy is interpreted by supposing the existence of a roughening transition around $1 \mathrm{~K}$ at the surface of the crystals.

More important are the recent measurements of $\alpha$ by Landau et al. [8] and by Kechichev et al. [10]. Kechichev et al. have measured the dispersion relation for freezing-melting waves at the surface of helium crystals. They seem never to observe any facets at the surface and find a unique value of $0.23 \mathrm{erg} . \mathrm{cm}^{-2}$ for $\alpha$ in the hcp-liquid case whatever the temperature [12]. Landau et al. [9] have observed with an optical technique that, below $1 \mathrm{~K}$ and not above, hcp He crystals are usually faceted during growth and stable facets sometimes remain at the equilibrium. They measure $\alpha$ by looking at the shape of the main part of the meniscus which is rounded and again find a value $\left(0.16 \mathrm{erg} . \mathrm{cm}^{-2}\right)$ which is constant in temperature. The latter experiment has suggested to us a new interpretation of BEL's experiment.

Kechichev's and especially Landau's values for $\alpha$ are in good agreement with BEL's values above $1 \mathrm{~K}$. We think therefore that the measurement in the bubble experiment is valid in this temperature range. We suppose then that the crystal undergoes a roughening transition at $1 \mathrm{~K}$, facets appearing at the interface. perpendicular to some high symmetry axis (c or a). As noted by Landau et al. [9], this does not usually concern large interfaces where the gravity plays an important role. But on a small hemispherical bubble controlled by surface tension, where all the crystalline directions are present, a few facets should exist. Laplace's law (1) must be rewritten as :

$$
\Delta p=\left(\alpha+\alpha_{1}^{\prime \prime}\right) \frac{1}{R_{1}}+\left(\alpha+\alpha_{2}^{\prime \prime}\right) \frac{1}{R_{2}}
$$

where $R_{1,2}$ are the radii of curvature in the two directions which diagonalize the tensor $\frac{\partial^{2} \alpha}{\partial \varphi_{i} \partial \varphi_{j}}$ of the second derivatives of $\alpha$ with respect to the crystallographic angles $\varphi_{i}[13]$

$$
\left(\alpha_{i}^{\prime \prime}=\left(\frac{\partial^{2} \alpha}{\partial \varphi_{i}^{2}}\right)\right) .
$$

In the case of a faceted crystal, one knows from Wulf's theorem [14] that each facet in a direction $\varphi_{0}$ corresponds to a cusp in the polar plot of $\alpha(\varphi)$ in the same direction $\varphi_{0}$. At this point one can write :

$$
\left.\frac{\partial \alpha}{\partial \varphi}\right|_{\varphi_{0}} ^{ \pm}= \pm \frac{E_{\mathrm{s}}}{a}
$$

where $E_{\mathrm{s}}$ is the energy per unit length of a step of height $a$ on a high symmetry plane face with normal direction $\varphi_{0}$. The \pm sign refers to the two derivatives on the $\left(\varphi>\varphi_{0}\right)$ and $\left(\varphi<\varphi_{0}\right)$ sides of the cusp. The jump of $\partial \alpha / \partial \varphi$ at $\varphi_{0}$ implies an infinite value for $\alpha^{\prime \prime}$ and an infinite radius of curvature, that is a facet.

However, this is not enough to understand why BEL's measurement gives such a high value of $\alpha$ below $1 \mathrm{~K}$. Namely the condition for the stability of the bubble is not significantly changed by the appearance of facets, as long as there remain on it some rounded parts.

We suppose that the hole is completely blocked by a unique facet. As this hole is $1 \mathrm{~mm}$ thick and has a diameter of $2 \mathrm{~mm}$, this is the lowest energy configuration for a large range of crystal orientations (a or c $0^{\circ}$ to $30^{\circ}$ from the vertical). In order for the crystal to go through it has to grow with the aid of screw dislocations emerging on the facet. We suppose that 
the steps between atomic planes at the surface of the facet are attached to two screw dislocations with opposite sign. In the presence of a given difference of pressure $\Delta p$ between the two sides of the facet, the steps are bent. They become unstable if $\Delta p$ is larger than a critical value for which the step is a semicircle, leading then to the growth of the crystal. In our case the minimum $p$ for crystal growth is obtained for steps with length $2 R$, where $R$ is the hole radius and it is simply given by :

$$
\Delta p=\frac{E_{\mathrm{s}}}{a R}
$$

where $E_{\mathrm{s}}$ and $a$ are quantities which already appeared in eq. (3). The condition for the stability of the facet in BEL's experiment is then

$$
\Delta \rho g H \leqslant \frac{E_{\mathrm{s}}}{a R} .
$$

In other words, the value $1 \mathrm{erg} . \mathrm{cm}^{-2}$ measured by BEL below $1 \mathrm{~K}$ is not that of $\alpha$ but that of

$$
\frac{1}{2} \frac{E_{\mathrm{s}}}{a}=\frac{1}{2} \frac{\partial \alpha}{\partial \varphi}\left(\varphi_{0}\right) .
$$

This probably leads to a very sharp cusp in the polar plot of $\alpha(\varphi)$ at $\varphi_{0}$ : if one assumes

$$
\alpha\left(\varphi_{0}\right) \simeq\langle\alpha\rangle \simeq 0.14 \mathrm{erg} . \mathrm{cm}^{-2}
$$

(the $\langle>$ denotes an average over $\varphi$ ) and if one uses $E_{\mathrm{s}} / a=2 \mathrm{erg} \cdot \mathrm{cm}^{-2}$, half the angle of the cusp is $0.14 / 2 \mathrm{rad} .=4^{\circ}$. Let us remark that in BEL's experiment, the measured value changes from $\alpha$ to $\frac{1}{2} \frac{E_{\mathrm{s}}}{a}$ at a temperature such that $\frac{1}{2} \frac{E_{\mathrm{s}}}{a}$ becomes larger than $\alpha$, not at the exact roughening transition temperature which might be slightly above, and where $E_{\mathrm{s}}$ is strictly zero. Let us remark in addition that the existence of a unique step crossing the hole is sufficient for the crystal to grow in the way described above. There must be many shorter steps joining for example a screw dislocation to its nearest neighbour with opposite sign. The latter participate in the growth at larger $\Delta p$, as found in another experiment [15].

Andreev [11] has supposed that kinks on steps are quantum defects whose delocalization gives rise to a quantum roughening of the surface of helium crystals at absolute zero. The existence of a roughening transition at $1 \mathrm{~K}$, as proposed here, could seem purely classical and in complete disagreement with Andreev's theory. One could even have predicted the order of magnitude of the transition temperature $T_{\mathrm{R}}$ by using a very general and classical argument : in the theory of the roughening transition, $T_{\mathrm{R}}$ is always found to be of the order of $J$, the interaction energy between nearest neighbours. A rough estimate of $J$ is $a^{2} \alpha$. Taking $a \sim 3.5 \AA$ and $\alpha \sim 0.14$ erg $\mathrm{cm}^{-2}$ gives $T_{\mathrm{R}} \sim 1 \mathrm{~K}[16]$.

A more precise calculation is needed to know if the step energy is reduced by some quantum delocalization and the roughening transition consequently displaced, or if the system can be analysed in a purely classical way. Nevertheless, we do not forget that helium has two great advantages due to quantum mechanics which make it a very good candidate for the general study of crystal surfaces : the transport of mass and heat is accomplished by a superfluid, and the large zero point motion makes it difficult not to work with good quality single crystals.

Acknowledgments. - We are extremely grateful to the Haifa group who first observed the existence of facets at the surface of helium 4 crystals, and their disappearance around $1 \mathrm{~K}$, and specially to $\mathrm{J}$. Landau and Y. Avron for a very fruitful exchange of letters. We are indebted to D. O. Edwards who corrected some of our ideas, and to J. Vannimenus, G. Toulouse. A. F. Andreev, J. Lajzerowicz and A. Libchaber for many helpful discussions.

\section{References}

[1] Burton, W. K. and Cabrera, N., Discuss. Faraday Soc. 5 (1949) 33 and

Burton, W. K., Cabrera, N. and Franck, F. C., Philos. Trans. R. Soc., London 243 A (1951) 299.

[2] A fairly complete review can be found in :

Weeks, J. D. and Gilmer, G. H., Advances in Chemical Physics, edited by I. Prigogine and S. A. Rice, vol. 40, p. 157.

[3] Kosterlitz, J. M. and Thouless, D. J., J. Phys. C 6 (1973) 1181 and

Kosterlitz, J. M., J. Phys. C 7 (1974) 1046.

[4] See for example the review given in :

LeAMY, M. J., Gilmer, G. H. and JACKSON, K. A., in Surface Physics of Materials Blakely ed. (Ac. New York) 1975, vol. 1, p. 121-188.
[5] Bishop, D. J. and RePPY, J. D., Phys. Rev. Lett. 40 (1978) 1727.

[6] Halperin, B. I. and Nelson, D. R., Phys. Rev. Lett. 41 (1978) $121,519 \mathrm{E}$.

Young, A. P., Phys. Rev. B 19 (1979) 1855.

Morf, R. H., Phys. Rev. Lett. 43 (1979) 931.

[7] JACKson, K. A. and Miller, C. E., J. Crystal Growth 40 (1977) 169.

[8] Balibar, S., Edwards, D. O. and Laroche, C., Phys. Rev. Lett. 42 (1979) 782.

[9] Landau, J., Lipson, S. G., Maatanen, L. M., Balfour, L. S. and EDWARDS, D. O., to be published.

[10] KeChichev, K. O., PARshin, A. J. and Rabkin, A. V., J.E.T.P. Lett. 30 (1979) 63. 
[11] Andreev, A. F. and Parshin, A. Ya., Sov. Phys. J.E.T.P. 48(4) (1978) 763.

[12] Note added after submission of this letter. Very recent measurements by Kechichev et al. give values of $\alpha$ from $\sim 0.1$ to $\sim 0.2 \mathrm{erg} . \mathrm{cm}^{-2}$ depending on the crystal orientation. The disappearance of facets around $1 \mathrm{~K}$ has been observed in Moscow also. We are very grateful to them for communicating their results prior to publication.

[13] Herring, C., in Structure and Properties of Solid Surfaces, edited by R. Gomer and C. S. Smith (University of Chicago Press) 1953, p. 39.
[14] See for example :

BRICE, J. C., The Growth of Crystals from the Melt (North Holland Publishing Company, Amsterdam) 1965, p. 40

[15] Castaing, B., Balibar, S. and Laroche, C., to be published in J. Physique 41 (August 1980).

[16] More precise information is given by Y. Avron, including various inequalities between the respective transitions $T_{\mathrm{R}}$ of the different faces of the different crystals. Private communication, and to be published. 